Biglari, Amir \& Marc Bonhomme (éds). 2018. La Présupposition entre théorisation et mise en discours (Paris : Classiques Garnier)

Silvia Adler

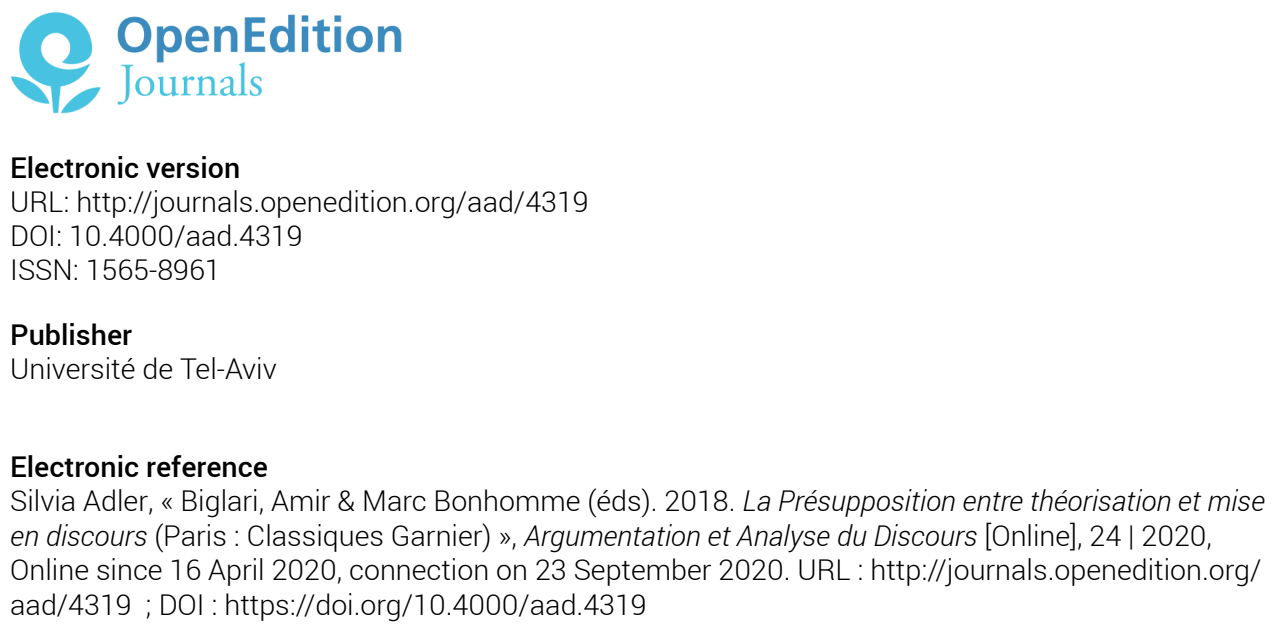

This text was automatically generated on 23 September 2020.

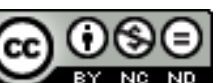

Argumentation \& analyse du discours est mis à disposition selon les termes de la licence Creative Commons Attribution - Pas d'Utilisation Commerciale - Pas de Modification 4.0 International. 


\title{
Biglari, Amir \& Marc Bonhomme (éds). 2018. La Présupposition entre théorisation et mise en discours (Paris : Classiques Garnier)
}

\author{
Silvia Adler
}

\section{REFERENCES}

Biglari, Amir \& Marc Bonhomme (éds). 2018. La Présupposition entre théorisation et mise en discours (Paris : Classiques Garnier), 582 pages. ISBN : 978-2-406-006646-0

1 A travers ses 22 contributions, et son ample introduction signée par les directeurs Amir Biglari et Marc Bonhomme, l'ouvrage collectif La Présupposition entre théorisation et mise en discours (2018) entreprend d'examiner la notion de présupposition par le prisme tant de ses enjeux théoriques que de ses applications discursives. Un tel ouvrage se justifie, selon les directeurs du recueil, du fait que la présupposition reste toujours un concept flou et instable, état résultant (a) d'une théorisation hétéroclite (approches logicistes, linguistiques, énonciatives, pragmatiques) ; (b) d'un vaste domaine d'application (en lexique, syntaxe, prosodie) ; (c) d'une indétermination définitoire, qui brouille les frontières entre la présupposition et des notions avoisinantes comme le sous-entendu et l'implicature. Un tel recueil s'impose d'autant plus que les recherches sur la présupposition tendent à négliger le discours oral et écrit en action, dans les méandres de ses productions concrètes et contextualisées. Aussi les auteurs se proposent-ils quatre objectifs de nature diverse.

2 Le premier objectif consiste à développer la théorisation sur la présupposition afin de mieux intégrer ses dimensions pragmatico-énonciatives. Le produit de cette revisite de la théorisation sera, entre autres, une clarification des rapports que la présupposition entretient avec les implicatures, les savoirs partagés, le sens commun ou l'idéologie, 
ainsi qu'une réévaluation des faits syntaxiques qui génèrent le présupposé. En deuxième lieu, à travers ses contributions d'ordre théorique, l'ouvrage explore divers aspects relatifs à l'analyse discursive de la présupposition, approche qui projette un éclairage édifiant sur les plans (a) macrosyntaxique, du fait que l'étude de la présupposition en contexte engendre une interrogation sur des phénomènes subsidiaires, dont, entre autres, la hiérarchisation des présupposés entre eux, et/ou entre eux et d'autres phénomènes avoisinants, tel le sous-entendu ; (b) interactif, puisqu'une approche discursive est à même de mettre en valeur la dimension dialogique de la présupposition; (c) rhétorique, du fait que l'étude de corpus authentiques promeut une vision de la présupposition comme stratégie argumentative. En troisième lieu, Biglari et Bonhomme, en parfait accord avec la ligne directrice marquée par bon nombre d'articles, identifient l'importance d'une analyse textuelle qui préconise l'authenticité et l'intégralité du texte, pour donner accès à la dimension intertextuelle de la présupposition, mais aussi pour mettre en évidence la réinterprétation ou la modification potentielle de celle-ci dans le texte. Finalement, l'ouvrage se fixe pour but d'amplifier le champ d'action de la présupposition pour l'appliquer au domaine de la sémiotique visuelle, extension qui permettra de problématiser la question de la pertinence et de l'utilité de la présupposition à l'étude du visuel ou des supports multimodaux.

3 Après l'introduction, le recueil se déploie en cinq parties : la première partie, avec ses six contributions - selon ordre d'apparition - de L. de Saussure, J. Moeschler, S. Deloor et J.-Cl. Anscombre, P.-Y. Raccah, J. Jayez et R. Reinecke, et G.-E. Sarfati, opère un cadrage théorique et conceptuel de la présupposition. En rappelant les propriétés essentielles de celle-ci, Louis de Saussure place la présupposition à la frontière entre sémantique et pragmatique. Il plaide pour une distinction entre la présupposition linguistique, qui se déclenche localement, stricto sensu, et la présupposition discursive, formée globalement, à partir de la totalité de l'énoncé - donc lato sensu - considérée comme une implicature faible d'arrière-plan qui ne vise pas à engendrer des inférences particulières, mais qui est néanmoins indispensable pour établir la pertinence aux circonstances de l'énonciation. Jacques Moeschler poursuit la question de la répartition de travail entre sémantique et pragmatique, pour mieux cerner les frontières équivoques entre présupposition et implicature. S'inscrivant dans une approche pragmatique formelle, son étude propose une réévaluation de la présupposition en tant que phénomène pragmatique, et par conséquent, une meilleure différentiation entre présupposition et implicature, fondée sur des critères complémentaires. Ainsi, des tests formels feront apparaître les présuppositions comme des informations implicites d'arrière-plan, non inférentielles, vériconditionnelles et impliquant l'engagement $\mathrm{du}$ locuteur, à l'encontre des implicatures, identifiées comme des informations implicites de premier plan, inférentielles, non véridiconditionnelles et n'impliquant pas l'engagement du locuteur, donc annulables.

4 Se développant à partir de la pragmatique de Ducrot, les études de Sandrine Deloor et de Jean-Claude Anscombre et de Pierre-Yves Raccah s'attardent sur les dimensions discursive et argumentative de la présupposition. Deloor et Anscombre défendent une approche énonciative de la présupposition, laquelle s'oppose à une optique référentialiste promouvant une vision sociolinguistique et s'appuyant sur des connaissances partagées, externes au discours. L'approche énonciative présente l'avantage de valoriser les représentations discursives au moment de l'énonciation, et peut par conséquent mieux rendre compte de phénomènes divers, dont l'unicité liée à 
l'article défini, unicité qui se motive désormais en rapport avec l'événement construit par l'énoncé contenant, et non à travers un rapport avec un contexte extralinguistique.

5 Pour sa part, Raccah dresse un bilan des théories vériconditionnelles de la présupposition pour enfin mettre en évidence le rôle que joue la présupposition dans la manipulation idéologique. En proposant une réorientation sémantique de la présupposition - à la lumière des faiblesses des théories vériconditionnelles - et en insistant sur la nécessité méthodologique de dégager les "champs topiques lexicaux " responsables de la gestion des "garants d'argumentation », la présupposition acquiert son pouvoir argumentatif et manipulatoire, du fait d'une construction de sens en situation et donc d'une influence successive sur les points de vue (croyances et valeurs). Jacques Jayez et Robert Reinecke, quant à eux, analysent les résultats d'une expérience comportementale utilisant des phrases complexes dites factives, qui présupposent la vérité de la proposition enchâssée (par exemple « savoir que $\mathrm{P}$, regretter que $\mathrm{P}$ »), pour détecter une différence éventuelle de saillance cognitive entre contenu principal et contenu présupposé.Les résultats de leur étude suggèrent que les propriétés discursives (suivant la loi d'enchaînement théorisée par Ducrot, 1972) sont un facteur de différenciation plus plausible que la simple saillance entre contenu posé principal et contenu présupposé enchâssé.

6 L'article qui clôture la première partie, celui de de Georges-Elia Sarfati, plaide pour une extension de la problématique présuppositionnelle, au profit d'une théorie de l'évidence sémantique, qui prend en compte la description des formations préénonciatives et énonciatives du sens commun, à partir d'une conceptualisation des états du discours. C'est la dimension linguistique du sens commun, plutôt que ses volets philosophiques ou sociologiques, qui est privilégié par Sarfati. L'auteur oriente les lecteurs vers une réflexion sémio-discursive du sens commun, en insistant sur ses quatre étapes de formation (canon, vulgate, doxa, idéologie) et ses variations discursives dynamiques. Cette étude explore aussi la place du sujet-acteur dans la production et la reproduction du sens commun, à partir des récents déploiements de la pragmatique topique.

7 A travers ses trois articles, la deuxième partie du recueil associe la présupposition à l'énonciation grammaticale. Les deux premières études - celle de Corinne Rossari, et celle de Mervi Helkkula - interrogent des modèles présuppositionnels syntaxiques ; la troisième - celle de Marc Bonhomme - examine la présupposition en rapport avec la norme grammaticale. En effectuant une étude sur corpus des séquences «certes/ d'accord/ oui, P, mais Q», Rossari se penche sur la présupposition discursive présupposition enclenchée au moment de l'énonciation - dans les structures concessives, et argue que le présupposé (l'amorce de la séquence concessive) et le posé (introduit par un marqueur comme "mais») sont indissociables: alors que le présupposé sert de background discursif, le posé contrastif enrichit ce background et s'impose comme central par le locuteur. Le présupposé concerne alors le relief cognitif de l'énonciation, plutôt que les propriétés sémantiques dans le domaine de la présupposition. Helkkula s'attaque aux constructions pseudo-clivées (« ce que/ ce qui..., c'est »). Cette construction présente le contenu isolé ou encadré comme une croyance commune aux interlocuteurs, voire un contenu indéniable, mais sert aussi d'agent de cohérence dans le discours, en ce qu'elle consolide l'enchaînement par rapport à un contexte anaphorique, en même temps qu'elle met en valeur le contraste par rapport au contenu cataphorique. Le recours à cette construction permet au locuteur d'assumer 
une position d'autorité, ou de contrecarrer des objections potentielles, d'où ses exploitations rhétoriques.

8 La dernière contribution de cette section est signée par Bonhomme, qui se propose d'éclairer le rôle important de la présupposition dans le discours grammatical normatif des remarqueurs $d u 17^{\mathrm{e}}$ siècle. Selon l'auteur, à la base du français classique, les remarques rédigées par Vaugelas, Ménage et Bouhours sur la langue française sont parcourues par des présupposés cadratifs qui imposent une idéologie préconstruite du bon usage, et préconisent le rejet de nombreuses variations langagières. En outre, les productions grammaticales des remarqueurs mettent en jeu des présupposés discursifs conjoncturels qui révèlent la gestion personnelle du bon usage - au-delà du fonds doctrinal commun - par chaque remarqueur, sur le plan de l'argumentation normalisante et de l'univers de croyances.

Les deux sections qui suivent regroupent des textes qui explorent le fonctionnement de la présupposition dans des discours de types divers. Si la troisième section porte spécifiquement sur la présupposition dans les discours littéraires (Marion Carel, Amir Biglari, Jean-Paul Dufiet, Anna Jaubert), la quatrième, avec ses six contributions (Catherine Kerbrat-Orecchioni, Ruggero Druetta, Paola Paissa, Ruth Amossy, Alain Rabatel, Karine Berthelot-Guiet) traite des textes politico-médiatiques.

10 En tête de la troisième section, l'analyse d'un poème de guerre entreprise par Carel montre l'interdépendance entre la matière présupposée et la matière posée. Loin de constituer un simple préalable à une assertion, la présupposition complète sémantiquement l'énoncé, tout en influençant la construction textuelle et l'organisation discursive. L'auteure distingue deux sortes de présupposés: les présupposés argumentatifs, qui forment avec leurs posés un unique enchaînement argumentatif (" présupposé donc posé/ présupposé pourtant posé »), et les présupposés co-signifiés, qui constituent avec leurs posés deux facettes d'un même "Événement ». Biglari étudie les effets manipulatoires de la présupposition dans Les Contemplations de Victor Hugo par l'intermédiaire de deux modalités : le "faire croire» et le "faire éprouver ». Cette contribution met en évidence le fait qu'en présentant un cadre discursif comme déjà connu et partagé, donc comme allant de soi, la présupposition s'impose au lecteur. La présupposition devient ainsi une véritable manipulation discursive. En outre, un effet semblable est à même de se produire par les éléments posés, sous certaines conditions.

11 Un corpus de textes dramatiques contemporains permettra à Dufiet d'identifier les caractéristiques discursives du présupposé. Son analyse sémio-linguistique et pragmatique mettra en valeur les rapports entre le posé et le présupposé et la manière dont ceux-ci nourrissent la narration théâtrale. En outre, étant donné son rôle clé dans la relation référentielle, ses particularités énonciatives, mais aussi son rôle important dans la dialogie externe du texte dramatique, le présupposé constitue également une structure féconde du dialogue théâtral et de l'interaction théâtrale en général.

Cette section se clôt par la contribution de Jaubert. L'auteure part de l'opposition entre présupposition et sous-entendu (Ducrot 1969-1978), selon laquelle, pour la présupposition, l'implicite est linguistiquement marqué, indépendant des circonstances de l'énonciation, alors que pour le sous-entendu, l'implicite est livré aux aléas du contexte. A travers une étude sur corpus - le dialogue théâtral de Marivaux - Jaubert établit que la frontière entre les processus discursifs inférentiels est en réalité moins tranchée qu'on ne le pense. Cette étude défend une thèse continuiste, une transition 
graduelle, ou une optique modulaire, révélant imbrications et enchaînements entre les deux stratégies-phares de l'implicite.

En début de la section suivante, Kerbrat-Orecchioni considère la présupposition du point de vue de son fonctionnement in situ, à partir d'un corpus de débats présidentiels télévisés représentant plus de 14 heures d'enregistrement. L'auteure soulève la question de la pertinence de la distinction classique entre présupposé et sous-entendu à ce type d'observables, et met en valeur les principaux types de présupposés exploités dans ce type de discours dynamique. Son analyse permet de dégager le mécanisme argumentatif et stratégique des présupposés exploités par les débatteurs, mais aussi la manière dont ceux-ci sont négociés entre les locuteurs. Druetta s'intéresse, lui aussi, au débat politique. Son article montre que les énoncés parenthétiques, qui créent d'habitude une plateforme privilégiée pour la présupposition pragmatique, présentée comme partagée et consensuelle, correspondent, dans un discours médiatique agonal tel que le débat télévisé entre F. Hollande et N. Sarkozy, à un coup de force vis-à-vis de l'adversaire. La raison en est que cette stratégie, par l'intermédiaire de sa macrosyntaxe et de sa prosodie, soustrait les contenus des parenthèses à la possibilité de la réfutation, sous peine pour le rival d'afficher un comportement non coopératif, nuisible à son ethos.

14 Paissa approfondit davantage le regard sur le discours électoral. Son étude, qui examine les présupposés liés à la formule «majorité silencieuse ", illustre les enjeux discursifs de cette formule dans les campagnes présidentielles de Sarkozy de 2007 et 2012, en se focalisant sur des présupposés constitutifs et occasionnels. A travers une étude de réception, l'auteure met en évidence aussi les réactions des blogueurs - qui alternent entre le refus, l'accommodation et l'acceptation - envers les fondements présuppositionnels de cette formule.

15 A partir d'une micro-analyse de l'énoncé inaugural tiré d'un article de B.- H. Lévy publié en 2010 dans Le Point, Amossy s'interroge sur le fonctionnement argumentatif de la présupposition en contexte polémique. Même si elle est soustraite à la discussion, la présupposition n'en est pas moins destinée à un allocutaire qui, à son tour, peut l'adopter ou la refuser. Cependant, en situation de controverse, avance l'auteure, le rejet des présupposés signale la continuation du dialogue sur le mode du dissensus, plutôt que sa rupture (comme le voudrait Ducrot). Cette optique dialogique et communicationnelle de la présupposition permet à Amossy de conclure que la polémique s'exerce non seulement dans la confrontation des thèses, mais aussi dans le soubassement des interactions.

Rabatel développe des observations théoriques relatives au fonctionnement textuel de la présupposition. Il met en valeur la gradualité des relations entre présupposés et sous-entendus dans un article du Parisien, paru en 2014, suivant la nature lexicale ou syntaxique des présupposés, l'ordre des constituants, les régimes de lecture, la prise en compte des stratégies de mise en page et de mise en discours. L'auteur insiste sur le fait que ces derniers paramètres sont davantage cruciaux pour les sous-entendus que pour les présupposés, ce qui suggère que les pratiques d'implication ne sont pas dissociées d'enjeux déontologiques dans le discours de presse.

17 La contribution qui clôt cette partie étudie les discours publicitaires contemporains. D'après son auteure, Berthelot-Guiet, les présupposés que les discours publicitaires véhiculent sont rarement examinés. Aussi, cette étude aborde-t-elle la présupposition passée au filtre de la publicité suivant trois axes : (a) la présupposition au sens 
classique, dans le message publicitaire ; (b) la présupposition du produit, de la marque et de la consommation; (c) la présupposition métadiscursive, qui postule la reconnaissance du caractère publicitaire des discours et des tentatives investies par les marques afin de s'en démarquer.

Christophe Genin, qui s'interroge sur les conditions d'interprétation des images, ouvre le dernier volet de l'ouvrage, consacré à la sémiotique visuelle. À travers un travail comparatiste (France/Chine), cet auteur est amené à conclure que la compréhension d'images repose sur des prérequis culturels et que la conception de l'espace-temps préordonne nos conditions de réception. De là, il est amené à redéfinir le statut de la présupposition entre punctum originarium et regressus infinitum. Nicole EveraertDesmedt et Guy Everaert examinent la peinture figurative sous le prisme de la pragmatique néo-gricéenne. Plus précisément, les auteurs proposent d'adapter la notion de présupposition pour interpréter le tableau de Magritte, La Durée poignardée (1938). Selon eux, les glissements de perspective de cette peinture remettent en question le présupposé de l'unicité du point de vue et celui de la fixité du spectateur. Finalement, Georges Roque estime la pertinence de l'application de la présupposition linguistique aux images fixes. Dans un premier temps, cet auteur analyse des produits multimodaux, où la présupposition est transmise par le texte, plutôt que par l'image. Dans un second temps, il traite de la possibilité pour la syntaxe de l'image de construire à elle seule un présupposé, lorsque sur le plan narratif l'image renvoie à un moment antérieur, ce qui ramène à la question de la répartition du travail entre présupposé et sous-entendu.

19 S'adressant autant aux spécialistes qu'à des chercheurs venant d'horizons différents, dont l'intérêt touche aux questions de sens et de signification, en général, et aux défis soulevés tant par la question de la présupposition que par celle des concepts avoisinants, dont l'implicite et le sous-entendu, en particulier, La Présupposition entre théorisation et mise en discours s'impose comme un ouvrage de référence pour la recherche linguistique - théorique et pratique - actuelle. Par la richesse des contributions signées par des spécialistes de la présupposition ainsi que par des spécialistes de domaines variés qui ont examiné des corpus diversifiés sous le prisme de la présupposition, par les ponts jetés entre les diverses perspectives ou approches, ce recueil invite les lecteurs autant à la réflexion qu'au dialogue, et pose les jalons pour de futures recherches visant la dynamique de la construction de sens.

Outre la confrontation des corpus diversifiés ainsi que des méthodes adoptées par les différentes recherches, l'apport de cet ouvrage réside aussi dans la reconnaissance que la recherche sur la présupposition aura beaucoup à gagner si elle repose sur des supports authentiques et contextualisés, ce qui permettra de mettre en exergue la répartition de travail entre posé, présupposé et sous-entendu, et de montrer que les frontières entre ces concepts ne sont pas aussi nettes que le présente une grammaire décontextualisée qui a recours à des exemples artificiels. Le recueil accorde courageusement aussi une place à l'étude de la présupposition sur des supports multimodaux, en soi une entreprise louable.

Qu'on nous permette peut-être de regretter que le volet multimodal de la dernière partie soit un peu indécis ou inégalement traité. De plusieurs points de vue, il aurait pu compléter d'autres parties. En fait, du point de vue de l'organisation des chapitres, certaines contributions auraient pu mieux dialoguer avec d'autres si elles étaient rassemblées sous la même section (comme par exemple celles de P.-Y. Raccah et de R. 
Amossy ; l'étude de M. Carel qui aurait pu enrichir le chapitre contenant l'article de C. Rossari pour étayer la question du fonctionnement de la présupposition ; la contribution d'A. Rabatel, qui pourrait compléter la partie théorique, etc.). Mais ceci ne fragilise aucunement la valeur de ce recueil, qui, pour conclure, se présente comme un ouvrage fort riche et stimulant, tant par ses diverses approches pratiques que par les divers enjeux théoriques dont il est question.

\section{AUTHORS}

\section{SILVIA ADLER}

Université Bar Ilan, ADARR 